\title{
Bond Energies and Phosphate-Coordination of Magnesium Hydrate to Pyrimidine Nucleotide 5'- Monophosphates (CMP, UMP, dTMP) and NMR Shielding Tensors
}

\author{
M. Monajjemi", a, R. Sayyadia, G. Ghasemi ${ }^{\text {a }}$, Kh. Lalateh ${ }^{\text {a }}$, A. Nouria ${ }^{\text {a }}$, F. Naderi ${ }^{\text {a }}$ \\ ${ }^{a}$ Department of Chemistry, Islamic Azad University, Science and Research \\ Campus, Poonak, Hesarak, Tehran, Iran., P. O. Box:14515-775.
}

\begin{abstract}
:
The structure of model magnesium hydrate pyrimidine nucleotides (CMP, UMP, dTMP) adducts has been studied at the Hartree-Fock level and by using LANL2DZ basis set for magnesium and 6-31 $\mathrm{g}^{*}$ basis set for other atoms. The basis set superposition error (BSSE) begins to converge for used Method/basis set. A natural bond orbital calculation was carried out at the Hartree-Fock level and 6-31 $\mathrm{g}^{*}$ basis set of theory to determined donor-acceptor (bonding-antibonding) interactions. The gauge-invariant atomic orbital (GIAO) method and the continuous-set-of-gauge-transformation (CSGT) procedure were employed to calculate isotropic atomic shielding of the nucleotides and magnesium hydrate-pyrimidine nucleotides by using density functional theory at the B3LYP/6-31 $\mathrm{g}^{* *}$ and $\mathrm{HF} / 6-31 \mathrm{~g}^{* *}$ level.
\end{abstract}

Keywords: Ab initio Calculation, NMR shielding Tensor, Nucleotide 5'- Monophosphates, Magnesium Complexes.

\section{INTRODUCTION}

Magnesium, a divalent metal ion usually found in relatively high concentrations in vivo, stabilizes both DNA and RNA against thermal denaturation and in many cases is required for these molecules to function properly $/ 1,2,3,4 /$.

A nucleotide consists of three main subunits, the nucleobase residue (purine or pyrimidine), the sugar part and the phosphate group(s). The structures of the three common pyrimidine-nucleoside 5-monophosphates are shown in Fig $1 / 5,6,7,8$.

Nucleotide ligands offer a variety of potential binding sites for metal ions. In addition, these ligand molecules can adopt various conformations and thus exhibit complex steric properties. The whole area of

"e-mail:m_monajjemi@yahoo. com 
metal ion-nucleotide interactions is therefore of interest also from the point of general coordination chemistry. Main-group metal ions apparently bind only to the phosphates groups of nucleotides, whereas transition metal ions bind to the organic base as well $/ 9,10 /$, forming chelate complexes. The structurally characterized metal complexes of mononucleotides involve phosphate monoesters that carry two negative charges per phosphate group, in the normal protonation state consequently the role of the phosphate groups is either through direct metal binding or through electrostatic interaction with the metal. In solution, structures, of metal-nucleotide complexes have been studied mainly by NMR methods $/ 1,2 /$.

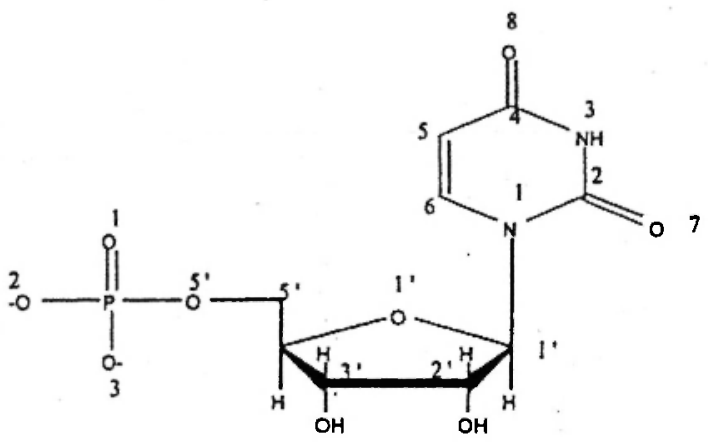

Uridines'-monophosphates(UM P 2-)

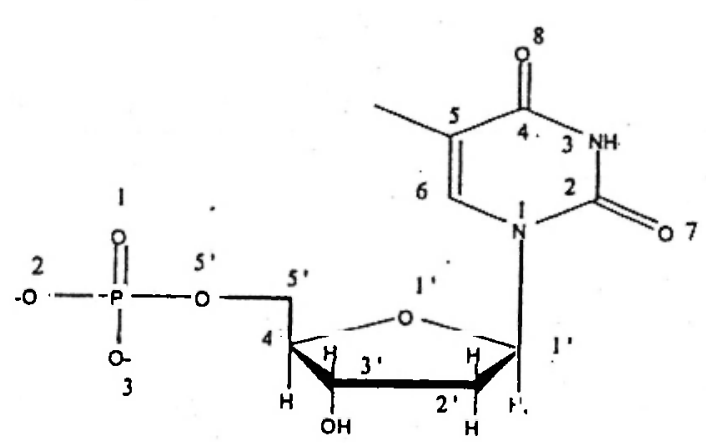

Thymine 5'-monophosphates(dTM P2-)

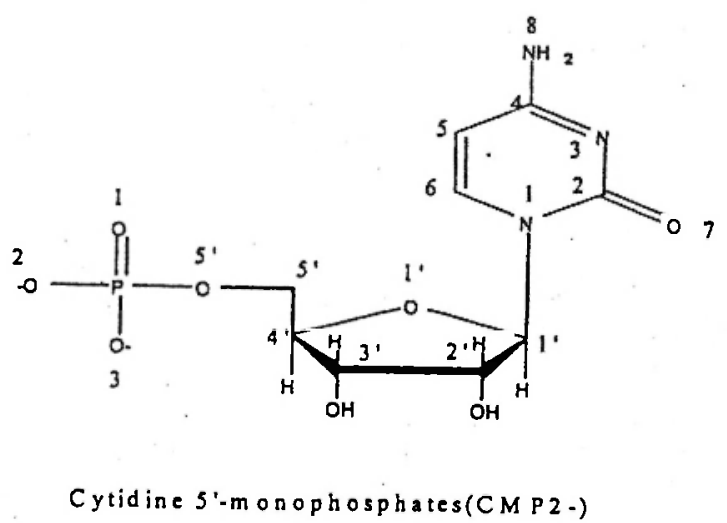

Fig. 1: Chemical structures of the pyrimidine-nucleoside 5'-monophosphates (UMP, dTMP, CMP).

Nuclear magnetic resonance techniques applied to poly- or mononucleotides can be directly used to detect the effects caused by the binding of a metal ion on these molecules in aqueous solutions. The magnetic resonance of protons has been most widely used. Depending on the nature of the metal in the complex, it will be convenient to measure one or another NMR parameter /15/. Moreover, the main NMR spectroscopic parameters are dependent on the second-order molecular property tensors $/ 16 /$. The NMR shielding tensor $\sigma$

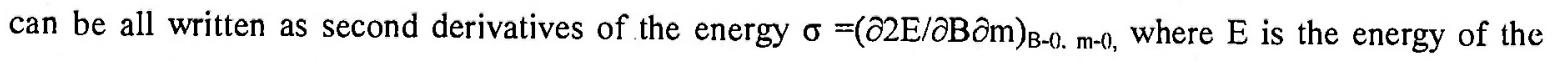
molecule, $B$ external magnetic field, $E$ electric intensity, and $n, m$ nuclear magnetic moments. Scalar parameter and $\sigma$ observable for an isotropic medium are defined as $1 / 3$ of the traces of the tensors $J$ and $\sigma$, respectively $/ 13 /$.

All of the optimized structure calculations were carried out at the HF/6-31g* level. Electronic structures 
were analyzed with the natural bond order method (NBO). In the NBO method, for optimally transforming a given wave function into localized form, corresponding to the one-center (lone pair) and twocenter (bond) elements of the chemist's Lewis structure picture. This is carried out by examining all possible interactions between filled (donor) Lewis type NBOs and empty (acceptor) non Lewis NBOs and estimating their energetic importance by two second-order perturbation theory. Since these interactions lead to loss of occupancy from the localized NBOs of the idealized Lewis structure into the empty non-Lewis orbitals (and thus, to departures from the idealized Lewis structure description), they are referred to as delocalization corrections to the zeroth-order natural Lewis structure.

In this study, structures of magnesium hydrate complexes of pyrimidine nucleotide 5'-monophosphate have been investigated by using $a b$ initio quantum-chemical calculation at the Hartree-Fock (HF) theoretical level with 6-31g* basis set for predicted polarization effects of the magnesium hydrate at the phosphate oxygen atom coordination. LANL2DZ for $\mathrm{Mg}$ and pyrimidine nucleotide 5'-monophosphates have been carried out. Hydrogen bonding will be discussed in term of observable properties such as geometry. The structures was supported by comparing the measured ${ }^{1} \mathrm{H}$-NMR spectra to the results of ab initio gaugeinvariant atomic orbital (GIAO) /15/ and continuous-set-of-gauge-transformation (CSGT) /16/ computations of chemical shifts. $A b$ initio calculations were compared using the experimental geometry, based on the structures derived from $\mathrm{X}$-ray diffraction data for nucleotide 5 '-monophosphaes. The basis set superposition error (BSSE) was computed, through the counterpoise method /17/ implemented in the Gaussian code 98 , for the most stable complexes.

NBO analysis is based on a method for optimally transforming a given wave function into localized form, corresponding to the one-center (lone pair) and two center (bond) elements of the chemist's Lewis structure picture $/ 18 /$.

\section{METHOD}

The structures of all the systems were optimized using the framework of the Hartree- Fock and LANL2DZ basis set for magnesium and 6-31g* basis set $/ 19,20$ / for other atoms. A natural bond orbital (NBO /18/ analysis was performed to the second-order perturbative estimates of donor-acceptor (bondingantibonding) interactions.

NMR analysis was performed using 6-31 $\mathrm{g}^{* *}$ basis set and the B3LYP /20-25/ level. The GIAO /15/ and CSGT /16/ methods were used to calculate the isotropic NMR shielding at the B3LYP/6-31g** and HF/9$31 \mathrm{~g}^{* *}$ of theory.

The interaction energies of the counterparts were estimated as the energy difference between the complex and the isolated components and were corrected for the basis set superposition error (BSSE). The BoysBernardi counterpoise method $/ 26 /$, applied at the magnesium hydrate nucleotide complexes geometry, is used to account for BSSE. According to this method:

$$
E_{\text {Ciorr. }}=E_{\text {Interaction }}+\Delta E_{\text {BSSSE }}
$$


where $E_{C o r r}$ is corrected-interaction energies, and:

$$
\begin{aligned}
\Delta E_{B S S E}= & {\left[E^{*} \text { Magnesium Hydrate }-E_{\text {Magnesium Hydrate }}(\text { Complex })\right]+} \\
& {\left[E^{*}{ }_{\text {lyrimidine nucleotide }}-E_{\text {Pyrimidine nucleotide }}(\text { Complex })\right] }
\end{aligned}
$$

where $\mathrm{E}^{*}$ indicates that the energy of components at complex geometry is calculated from Methods/Basis set of complex geometry.

\section{RESULTS}

The fully optimized molecular structures for conformations of magnesium hydrate-pyrimidine nucleotides at the HF/6-31g*:LANL2DZ is shown in Figure 2 and calculated optimized geometries are listed in Table 1:

\section{Table 1}

Optimized bond length $\left(A^{*}\right)$ of pyrimidine nucleotide complexes in the 6-31g*:LANL2DZ basis set

\begin{tabular}{|l|l|l|l|}
\hline Bond Length & Mg(H2O)4CMP & Mg(H2O)5UMP & Mg(H2O)5dTMP \\
\hline Mg-O(H2O) & $2.105,2.112$, & $2.138,2.170$, & $2.161,2.122$, \\
& $2.068,2.099$ & $2.141,2.149,2.096$ & 2.126, \\
Mg-O(PO4) & 1.923 & 1.942 & $2.120,2.155$ \\
O(PO4)---H(H2O) & 1.643 & $1.677,1.722$ & 1.952 \\
Ol'---H(H2O) & 1.952 & $1.981,2.592$ & 1.632 \\
O7---H(H2O) & 1.926 & & \\
N3----H(H2O) & 2.129 & & \\
P-O1 & 1.530 & 1.526 & 1.476 \\
P-O3 & 1.462 & 1.462 & 1.526 \\
P-O2 & 1.550 & 1.557 & 1.565 \\
\hline
\end{tabular}

Hard Lewis acids, including alkali, alkaline earth, and rare earth metal ions, display almost equal affinities to all the nucleoside monophosphates studied, suggesting that coordination takes place at the phosphate dianion without involvement of the base moiety.

Sigel $/ 17 /$ has shown, by comparing the stabilities of metal ion complexes of nucleoside monophosphates and simple phosphate monoesters, that the base moiety does not play any role in the complexing of CMP, UMP, dTMP. In other words, the complex stabilities are solely determined by the basicity of the phosphatc group and the metal ion affinity of the phosphate group.

Interaction with the phosphate group in the magnesium purine nucleotides was suggested to be either a direct one or hydrogen bonding to the 5 '-phosphate through a molecule of water $/ 28,29 \%$.

In the magnesium hydrate complexes of pyrimidine nucleotides the four and five $\mathrm{H} 2 \mathrm{O}$ ligands are coordinated to the magnesium cation, which is then bonded to the oxygen atom of the phosphate group at 
distances ranging between 1.912 and $2.150 \AA$, in agreement with the available experimental results ( 2 . $02 \mathrm{~A}^{\circ}$ and $2.11 \mathrm{~A}^{\circ}$ ) $/ 30 /$.

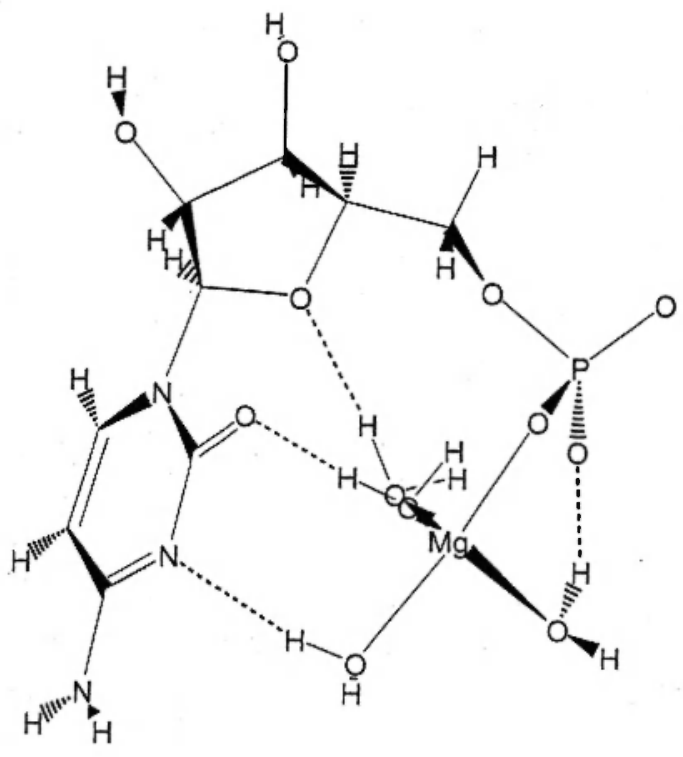

$\mathrm{HFMg}(\mathrm{H} 2 \mathrm{O}) 4 \mathrm{CMP}$

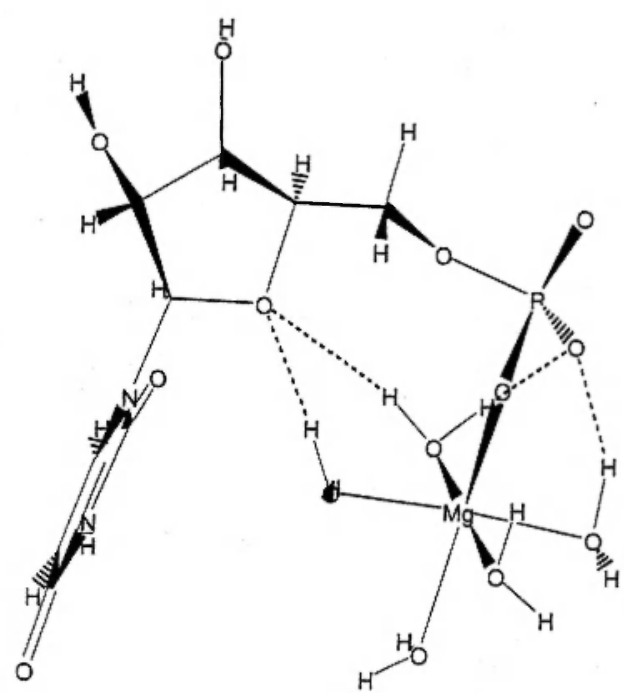

$\mathrm{HFMg}(\mathrm{H} 2 \mathrm{O}) 5 \mathrm{UMP}$

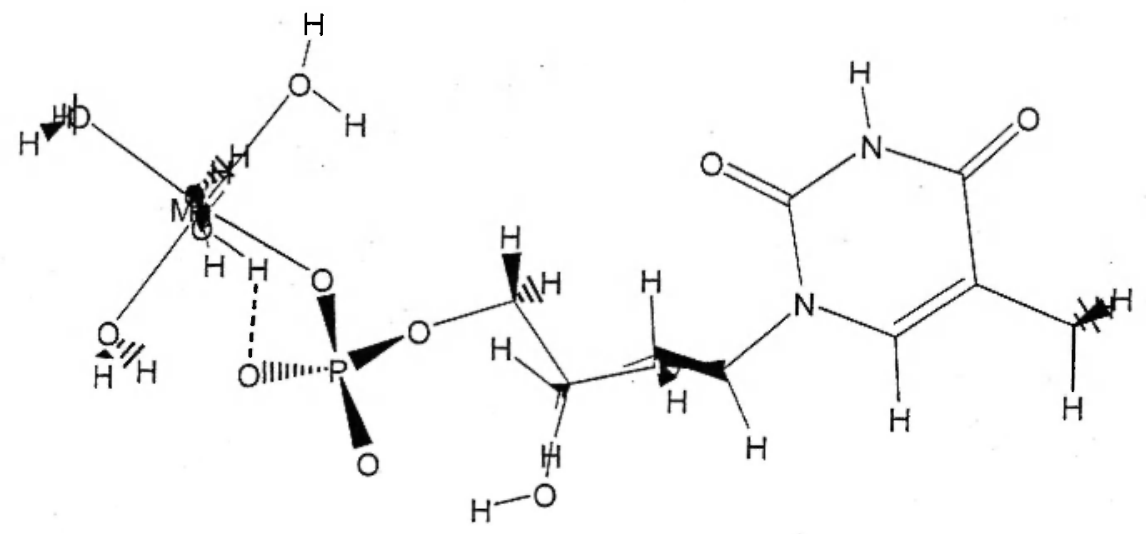

$\operatorname{HFMg}(\mathrm{H} 2 \mathrm{O}) 5 \mathrm{dTMP}$

Fig. 2: Optimized structures of Mg-nucleotide complexes in the HF/6-31g*:LANL2DZ Indirect (outersphere or hydrogen bonding) phosphate coordination probably occure between atoms oxygen phosphate atom and water molecules of Mgcoordination.

The conformation of the sugar ring in nucleotides and nucleosides can be examined by using a concept of pseudorotation, which utilizes a quantitative description of puckering and conformation in terms of the maximum torsion angle $\left(\tau_{m}\right)$ and the "Phase angle" of pseudorotation $(P)$, which is a function of the interrelationship between the five torsion angles $(\tau 0-\tau 4)$ in the nonplanar five-membered ring $/ 31 /$. The phase 
angle, $P$, and the maximum pucker, $\tau_{m}$, are calculated with eq 1 and $2 / 31 /$.

$$
\begin{aligned}
& \tan P=\left(\tau_{4}+\tau_{1}\right)-\left(\tau_{3}+\tau_{0}\right) / 2 \tau_{2}(\sin 36+\sin 72) \\
& \tau_{2}=\tau_{m} \cos P
\end{aligned}
$$

All the possible conformations are grouped into two categories, type $\mathrm{N}\left(\mathrm{P}=0 \pm 90^{\circ}\right)$ or $3^{\prime}$ endo and type $\mathrm{S}(\mathrm{P}=180 \pm 90 \div)$ or $2^{\prime}$ endo/ $\mathrm{Cl}$ ' exo $/ 32-34 /$. We have theoretically computed the $\mathbf{P}$ values for all nucleotides using HF/6-31g* level on the optimized structures. (Table 2) Pictorial representation of the anti and syn conformational ranges, defined by the torsion angle $\chi_{\mathrm{CN}}$ about the glycosidic bond $\mathrm{C} 1{ }^{1}-\mathrm{N} 1$, is defined in terms of the four nucleoside atoms $\mathrm{C} 2-\mathrm{N} 1-\mathrm{C} 1^{\prime}-\mathrm{O} 1^{\prime} / 35,38,39 /$

Table 2

\begin{tabular}{|c|c|c|c|}
\hline Dihedral Angles & $\mathrm{Mg}(\mathrm{H} 2 \mathrm{O}) 4 \mathrm{CMP}$ & $\mathrm{Mg}(\mathrm{H} 2 \mathrm{O}) 5 \mathrm{UMP}$ & $\mathrm{Mg}(\mathrm{H} 2 \mathrm{O}) \mathrm{dTMP}$ \\
\hline $\mathrm{MgOPO}^{\prime}$ & 83. 0 & 76.7 & 110.1 \\
\hline $\mathrm{PO}^{\prime} \mathrm{C} 5{ }^{\prime} \mathrm{C} 4$ & -155.7 & -162.6 & 60.5 \\
\hline $\mathrm{O}^{\prime} \mathrm{C} 5^{\prime} \mathrm{C} 4^{\prime} \mathrm{C} 3^{\prime}(\psi)$ & 164.7 & 179.1 & -88.4 \\
\hline O1'C1'N1C6 & -117.0 & -110.0 & -109.8 \\
\hline $\mathrm{O} 1^{\prime} \mathrm{C} 1 \mathrm{~N} 1 \mathrm{C} 2$ & 56.2 & 66.3 & 61.4 \\
\hline $\mathrm{O} 5^{\prime} \mathrm{C} 5^{\prime} \mathrm{C} 4^{\prime} \mathrm{O} 1^{\prime}$ & 48.5 & 62.6 & 152.7 \\
\hline $\mathrm{C}^{\prime} \mathrm{C} 4^{\prime} \mathrm{O} 1^{\prime} \mathrm{Cl}^{\prime}\left(\tau_{4}\right)$ & 32.2 & 38.7 & 70.0 \\
\hline $\mathrm{C} 3^{\prime} \mathrm{C} 21^{\prime} \mathrm{C} 1^{\prime} \mathrm{O} 1^{\prime}\left(\tau_{1}\right)$ & 8.6 & 2.4 & 28.1 \\
\hline $\mathrm{O}^{\prime} \mathrm{C} 4^{\prime} \mathrm{C} 3^{\prime} \mathrm{C} 2{ }^{\prime}\left(\tau_{3}\right)$ & 24.7 & -35.0 & 11.0 \\
\hline $\mathrm{C}^{\prime} \mathrm{O} 1^{\prime} \mathrm{Cl} 1^{\prime} \mathrm{C} 2^{\prime}\left(\tau_{0}\right)$ & -26.2 & -25.9 & -22.4 \\
\hline $\mathrm{C}^{\prime} \mathrm{C} 3^{\prime} \mathrm{C} 2^{\prime} \mathrm{Cl} l^{\prime}\left(\tau_{2}\right)$ & 10.0 & 19.8 & -23.0 \\
\hline
\end{tabular}

Dihedral Angles Complexes at 6-31g* Basis Set

Table 3

P Angles Complexes in $\mathrm{HF} / 6-31 \mathrm{~g}^{*}$ Level

\begin{tabular}{|c|c|}
\hline Complexes & P Angle \\
\hline$M g(\mathrm{H} 2 \mathrm{O}) 4 \mathrm{CMP}$ & 3.0 \\
\hline $\mathrm{Mg}(\mathrm{H} 2 \mathrm{O}) 5 \mathrm{UMP}$ & 1.7 \\
\hline $\mathrm{Mg}(\mathrm{H} 2 \mathrm{O}) 5 \mathrm{dTMP}$ & -1.5 \\
\hline
\end{tabular}

The results of these calculations showed that metal-nucleotide sugar conformations fall into categories C3' endo, syn.

The computed stabilization energies of the complexes for no-metalated and metalated pyrimidine 
nucleotides are compared by HF/6-31g* method (Table 4). Their relative stabilities are markedly influenced by the metalation. Metalation of pyrimidine nucleotides has been found to give more stability than in the nonmetalated molecule.

Table 4

Relative Energies (Hartree) for Mg-Pyrimidine Nucleotide Complexes in Gas Phase with HF/6-31g* Level

\begin{tabular}{|c|c|}
\hline Complexes HF/6 & 31g* \\
\hline CMP & 1450.764 \\
\hline UMP & 1470.623 \\
\hline dTMP & 1434.751 \\
\hline$M g(H 2 O) 4 C M P$ & 1954.752 \\
\hline$M g(H 2 O) 5 U M P$ & 2050.633 \\
\hline$M g(H 2 O) 5 d T M P$ & 2014.823 \\
\hline
\end{tabular}

Table 5 shows the interaction energies of metalated pyrimidine nucleotides. The increase in interaction energies of the metalated pyrimidine nucleotides is due to hydrogen bonding in metalated pyrimidine nucleotides (Table 1). Table 5 shows the value of BSSE and $\mathrm{E}_{\mathrm{l}+\mathrm{BSSE}}$ for the structures. Clearly for the all complexes, values of BSSE are rather small. Therefore, for these cases BSSE is negligible.

Table 5

$\mathrm{E}_{\mathrm{l}}$ (Interaction Energies), BSSE and E I+BSSE (Hartree) for Complexes in HF/6-31g* Level

\begin{tabular}{|l|c|c|c|}
\hline Complexes & $\mathbf{E}_{\mathrm{I}}$ & BSSE & $\mathbf{E}_{\mathrm{I}+\mathrm{BSSE}}$ \\
\hline $\mathrm{Mg}(\mathrm{H} 2 \mathrm{O}) 4 \mathrm{CMP}$ & -0.36075 & -0.0188594 & -0.3796106 \\
$\mathrm{Mg}(\mathrm{H} 2 \mathrm{O}) 5 \mathrm{UMP}$ & -0.36299 & 0.0282317 & -0.3347583 \\
$\mathrm{Mg}(\mathrm{H} 2 \mathrm{O}) 5 \mathrm{dTMP}$ & -0.37151 & -0.0111472 & -0.3826603 \\
\hline
\end{tabular}

NBO calculation shows the $\pi$-bonding contribution in the base moiety of nucleotides (Table 6). Strong electron delocalization in a best Lewis structure will also show up as donor-acceptor interactions. Table 7 shows the interactions that give the strongest stabilization.

The Lewis NBOs in Table 8 describe percentage of the total density, with the remaining non-Lewis density found primarily in the valence-shell antibonding. Also, analysis of the atomic charges is done by the natural bonding orbital (NBO) method. It was found that the charge on $\mathrm{O} 2$ in metalated pyrimidine nucleotides is higher than in nonmetalated pyrimidine nucleotides ( $\mathrm{O} 2$ coordinated to $\mathrm{Mg}$ ) (Table 9). As shown above, metalation of the pyrimidine nucleotides strongly influences their electronic structure, and this leads to energetic stabilization of the structures. There for, the increasing $\mathrm{O} 2$ basicity of the metalated nucleotides can be attributed to the relative stabilization and increased stability of $\mathrm{Mg}$-nucleotide complexes. 
Table 6

Hybridation Coefficient Of Bonds Calculated By NBO Method in HF/6-31g* Level

\begin{tabular}{|c|c|c|}
\hline Bond & $\mathrm{Mg}(\mathrm{H} 2 \mathrm{O}) 4 \mathrm{CMP}$ & $\mathbf{M g}(\mathbf{H 2 O}) 5 \mathrm{UMP}$ \\
\hline $\mathrm{P}-\mathrm{O} 2$ & $\sigma 0.4426\left(\mathrm{SP}^{3.02}\right) \mathrm{P}+0.8967\left(\mathrm{SP}^{1.77}\right) \mathrm{O} 2$ & $0.4360\left(\mathrm{SP}^{3.03}\right) \mathrm{P}+0.8999\left(\mathrm{SP}^{1.55}\right) \mathrm{O} 2$ \\
\hline $\mathrm{C} 2-\mathrm{O} 7$ & $\begin{array}{l}\sigma 0.5865\left(\mathrm{SP}^{2.03}\right) \mathrm{C} 2+0.8100\left(\mathrm{SP}^{1.29}\right) \mathrm{O} 7 \\
\pi 0.5289\left(\mathrm{SP}^{1.00}\right) \mathrm{C} 2+0.8487\left(\mathrm{SP}^{1.00}\right) \mathrm{O} 7\end{array}$ & $\begin{array}{l}0.58821\left(\mathrm{SP}^{1.87}\right) \mathrm{C} 2+0.8087\left(\mathrm{SP}^{1.26}\right) \mathrm{O} 7 \\
0.4907\left(\mathrm{P}^{1.00}\right) \mathrm{C} 2+0.8713\left(\mathrm{P}^{1.00}\right) \mathrm{O} 7\end{array}$ \\
\hline $\mathrm{C} 4-\mathrm{N} 3$ & $\begin{array}{l}\sigma 0.7655\left(\mathrm{SP}^{1.61}\right) \mathrm{N} 3+0.6434\left(\mathrm{SP}^{2.06}\right) \mathrm{C} 4 \\
\pi 0.8314\left(\mathrm{SP}^{1.00}\right) \mathrm{N} 3+0.55561\left(\mathrm{SP}^{1.00}\right) \mathrm{C} 4\end{array}$ & \\
\hline $\mathrm{C} 5-\mathrm{C} 6$ & $\sigma 0.7059\left(\mathrm{SP}^{1.79}\right) \mathrm{C} 5+0.7084\left(\mathrm{SP}^{1.51}\right) \mathrm{C} 6$ & $0.7025\left(\mathrm{SP}^{1.73}\right) \mathrm{C} 5+0.7117\left(\mathrm{SP}^{1.45}\right) \mathrm{C} 6$ \\
\hline & $\pi 0.7599\left(\mathrm{SP}^{1.00}\right) \mathrm{C} 5+0.6500\left(\mathrm{SP}^{1.00}\right) \mathrm{C} 6$ & $0.7613\left(\mathrm{SP}^{1.00}\right) \mathrm{C} 5+0.6484\left(\mathrm{SP}^{1.110}\right) \mathrm{C} 6$ \\
\hline $\mathrm{C} 4-\mathrm{O} 8$ & $\sigma$ & $0.5865\left(\mathrm{SP}^{1.195}\right) \mathrm{C} 4+0.8099\left(\mathrm{SP}^{1.20}\right) \mathrm{O} 8$ \\
\hline & $\Pi$ & $0.5259\left(\mathrm{SP}^{1.00}\right) \mathrm{C} 4+0.856\left(\mathrm{SP}^{1.00}\right) \mathrm{O} 8$ \\
\hline Bond & \multicolumn{2}{|l|}{$\mathrm{Mg}(\mathrm{H} 2 \mathrm{O}) 5 \mathrm{dTMP}$} \\
\hline $\begin{array}{l}\mathrm{P}-\mathrm{O} 2 \\
\mathrm{C} 2-\mathrm{O} 7\end{array}$ & $\begin{array}{l}\sigma 0.4452\left(\mathrm{SP}^{3.12}\right) \mathrm{P}+0.8954\left(\mathrm{SP}^{1.75}\right) \mathrm{O} 2 \\
\sigma 0.5912\left(\mathrm{SP}^{1.89}\right) \mathrm{C} 2+0.8065\left(\mathrm{SP}^{1.30}\right) \mathrm{O} 7 \\
\pi 0.5381\left(\mathrm{SP}^{1.00}\right) \mathrm{C} 2+0.8429\left(\mathrm{SP}^{1.00}\right) \mathrm{O} 7\end{array}$ & \\
\hline C4-N3 & $\begin{array}{l}\sigma \\
\pi\end{array}$ & \\
\hline $\begin{array}{l}\mathrm{C} 5-06 \\
\mathrm{C} 4-08\end{array}$ & $\begin{array}{l}\sigma 0.7079\left(\mathrm{SP}^{1.1 .}\right) \mathrm{C} 5+0.7063\left(\mathrm{SP}^{1.44}\right) \mathrm{O} 6 \\
\pi 0.7338\left(\mathrm{SP}^{1.00}\right) \mathrm{C} 5+0.6793\left(\mathrm{SP}^{1.00}\right) \mathrm{O} 6 \\
\sigma 0.5895\left(\mathrm{SP}^{2.05}\right) \mathrm{C} 4+0.8078\left(\mathrm{SP}^{1.25}\right) \mathrm{O} 8 \\
\pi 0.5575\left(\mathrm{SP}^{1.00}\right) \mathrm{C} 4+0.8302\left(\mathrm{SP}^{1.00}\right) \mathrm{O} 8\end{array}$ & \\
\hline
\end{tabular}

Table 7

The Stabilization Energy E(2) Associated with Delocalization for Interactions Giving the Strongest Stabilization in HF/6-31 $\mathrm{g}^{*}$ Level

\begin{tabular}{|c|c|c|c|}
\hline Complex & Donor NBO & Acceptor NBO & $\mathrm{E}(2) \mathrm{KCal} \mathrm{mol}^{-1}$ \\
\hline $\mathrm{CMP}$ & $\mathrm{LP}(3) \mathrm{O} 7$ & $\begin{array}{l}\mathrm{BD}^{*}(2) \mathrm{C} 2-\mathrm{N} 3 \\
\mathrm{BD}^{*}(1) \mathrm{C} 2-\mathrm{O} \\
\mathrm{BD}^{*}(1) \mathrm{C} 2-\mathrm{H}\end{array}$ & $\begin{array}{l}92.97 \\
2.96 \\
0.41\end{array}$ \\
\hline UMP & $\mathrm{LP}(3) \mathrm{O} 8$ & $\begin{array}{l}\mathrm{BD}^{*}(1) \mathrm{C} 4-\mathrm{C} 5 \\
\mathrm{BD}^{*}(2) \mathrm{C} 4-\mathrm{C} 5 \\
\mathrm{BD}^{*}(1) \mathrm{C} 4-\mathrm{O}\end{array}$ & $\begin{array}{l}1.37 \\
44.85 \\
1.69 \\
\end{array}$ \\
\hline dTMP & $\mathrm{LP}(3) \mathrm{O} 8$ & $\begin{array}{l}\mathrm{BD}^{*}(1) \mathrm{C} 4-\mathrm{C} 5 \\
\mathrm{BD}^{*}(2) \mathrm{C} 4-\mathrm{C} 5 \\
\mathrm{BD}^{*}(1) \mathrm{C} 4-\mathrm{O} \\
\mathrm{BD}^{*}(1) \mathrm{N} 8-\mathrm{C} 4\end{array}$ & $\begin{array}{l}0.26 \\
84.20 \\
4.51 \\
0.55 \\
\end{array}$ \\
\hline
\end{tabular}




\begin{tabular}{|c|c|c|c|}
\hline $\mathrm{Mg}(\mathrm{H} 2 \mathrm{O}) 4 \mathrm{CMP}$ & LP(1)N1 & $\begin{array}{l}\mathrm{BD}^{*}(2) \mathrm{C} 5-\mathrm{C} 6 \\
\mathrm{BD}^{*}(2) \mathrm{C} 2-\mathrm{O} 7 \\
\mathrm{BD}^{*}(1) \mathrm{C} 2^{\prime}-\mathrm{C} 1^{\prime} \\
\mathrm{BD}^{*}(1) \mathrm{C} 1^{\prime}-\mathrm{O} 1^{\prime}\end{array}$ & $\begin{array}{l}46.87 \\
53.45 \\
4.50 \\
7.87 \\
\end{array}$ \\
\hline $\mathrm{Mg}(\mathrm{H} 2 \mathrm{O}) 5 \mathrm{UMP}$ & LP(1)N1 & $\begin{array}{l}\mathrm{BD}^{*}(2) \mathrm{C} 5-\mathrm{C} 6 \\
\mathrm{BD}^{*}(2) \mathrm{C} 2-\mathrm{O} 7 \\
\mathrm{BD}^{*}(1) \mathrm{C} 2^{\prime}-\mathrm{C} 1^{\prime} \\
\mathrm{BD}^{*}(1) \mathrm{C} 1^{\prime}-\mathrm{O} 1^{\prime}\end{array}$ & $\begin{array}{l}52.40 \\
77.90 \\
5.29 \\
13.8 \\
\end{array}$ \\
\hline $\mathrm{Mg}(\mathrm{H} 2 \mathrm{O}) 5 \mathrm{dTMP}$ & LP(1)N1 & $\begin{array}{l}\mathrm{BD}^{*}(2) \mathrm{C} 4-\mathrm{C} 6 \\
\mathrm{BD}^{*}(2) \mathrm{C} 2-\mathrm{O} 7 \\
\mathrm{BD}^{*}(2) \mathrm{C} 2^{\prime}-\mathrm{C} 1^{\prime} \\
\mathrm{BD}^{*}(1) \mathrm{C} 1^{\prime}-\mathrm{O} 1^{\prime}\end{array}$ & $\begin{array}{l}37.11 \\
60.86 \\
3.85 \\
8.83 \\
\end{array}$ \\
\hline
\end{tabular}

Table 8

\%Total Lewis, Highest Energy Lewis NBO and Lowest Occupancy of Complexes in HF/6-31g* Level

\begin{tabular}{|l|c|c|c|}
\hline Complexes & \% Total Lewis & $\begin{array}{c}\text { Lowest occupancy } \\
(\mathbf{N} 1)\end{array}$ & $\begin{array}{c}\text { Highest energy } \\
\text { Lewis NBO (a.u) } \\
\text { (N1) }\end{array}$ \\
\hline $\mathrm{Mg}(\mathrm{H} 2 \mathrm{O}) 4 \mathrm{CMP}$ & 98.1650 & 1.58491 & -0.30486 \\
$\mathrm{Mg}(\mathrm{H} 2 \mathrm{O}) 5 \mathrm{UMP}$ & 98.2263 & 1.62492 & -0.25863 \\
$\mathrm{Mg}(\mathrm{H} 2 \mathrm{O}) 5 \mathrm{dTMP}$ & 98.5947 & 1.71447 & -0.42866 \\
\hline
\end{tabular}

Table 9

Natural Population of Phosphate Oxygen Atoms and P, Mg in Metalated Pyrimidine Nucleotides in HF/6-31g* Level

\begin{tabular}{|l|c|c|c|c|c|}
\hline Complexes & O1 & O2 & O3 & $\mathbf{P}$ & $\mathbf{M g}$ \\
\hline $\mathrm{Mg}(\mathrm{H} 2 \mathrm{O}) 4 \mathrm{CMP}$ & -1.20815 & -1.35704 & -1.12146 & 2.58446 & 1.73320 \\
\hline $\mathrm{Mg}(\mathrm{H} 2 \mathrm{O}) 5 \mathrm{UMP}$ & -1.31380 & -1.43884 & -1.23475 & 2.83431 & 1.81722 \\
\hline $\mathrm{Mg}(\mathrm{H} 2 \mathrm{O}) 5 \mathrm{dTMP}$ & -1.16998 & -1.35613 & -1.19831 & 2.61300 & 1.73812 \\
\hline
\end{tabular}

\section{NMR Chemical Shift Assignments}

The NMR shielding tensors were calculated using the gauge-including atomic orbital (GIAO) method and CSGT procedure by using B3LYP/6-31 $\mathrm{g}^{* *}$ and HF/6-31 $\mathrm{g}^{* *}$ level $/ 39 \%$. The isotropic part $\sigma$ iso of $\sigma$ is measured by taking the average of $\sigma$ with respect to the orientation to the magnetic field, $\mathrm{i}$. e., $\sigma$ iso $=(\sigma 11+\sigma 22+\sigma 33) / 3$. The results calculated are summarized in Tables 11-14).

The anisotropy is $\zeta=\mid \sigma 33-\sigma$ iso $\mid$, and the asymmetry is $\eta=(\sigma 22-\sigma 11) / \zeta / 40 \%$. 
$A b$ initio calculations yield the data in Tables 11,13 at B3LYP/6-31 $\mathrm{g}^{* *}$ method, showing that the values for the isotropic shielding $\mathrm{O} 2, \mathrm{O} 1, \mathrm{O} 3$ atoms in metalated CMP, UMP, dTMP are higher but the isotropic shielding of $\mathrm{C} 6$ atom is less than in nonmetalated CMP, UMP, dTMP. On the other hand, absolute isotropic TMS of C6, $\mathrm{H6}$ atoms in the metalated compounds is higher (Tables 12,14). It was found that the isotropic shielding $\mathrm{O} 8$ atom in metalated UMP, dTMP is less than in nonmetalated CMP, UMP, dTMP, but isotropic shielding 07 atom in metalated $\mathrm{CMP}$ was increased.

Table 10

Computed absolute isotropic shielding values for TMS

\begin{tabular}{|ll|c|c|}
\hline Method & ${ }^{13} \mathbf{C}$ & ${ }^{1} \mathbf{H}$ \\
\hline B3LYP/6-31g** & : GIAO & 191.8644 & 31.7532 \\
\hline B3LYP/6-31g** $\quad$ CSGT & 193.0751 & 29.3957 \\
\hline HF/6-31g** : GIAO & 203.1548 & 32.3360 \\
\hline
\end{tabular}

The results calculated by using HF/6-31g** level at GIAO to predict the NMR shielding tensor of oiso in Table 15, 16 show that the isotropic shielding of C6, H6 atoms in magnesium hydrate CMP, UMP, dTMP have been increased; on the other hand we have found a higher deshielding of the C6 and H6 to TMS in magnesium hydrate pyrimidine nucleotides than in the pyrimidine nucleotides. Isotropic shielding tensor of $\mathrm{N} 1, \mathrm{~N} 8, \mathrm{~N} 3, \mathrm{O} 7$ atoms in $\mathrm{Mg}(\mathrm{H} 2 \mathrm{O}) 4 \mathrm{CMP}$ and $\mathrm{N} 1, \mathrm{~N} 3, \mathrm{O} 7, \mathrm{O} 8$ atoms in $\mathrm{Mg}(\mathrm{H} 2 \mathrm{O}) 5 \mathrm{UMP}$ and $\mathrm{N} 1, \mathrm{~N} 3, \mathrm{O} 3$ atoms in $\mathrm{Mg}(\mathrm{H} 2 \mathrm{O}) 5 \mathrm{dTMP}$ have been decreased as compared to the CMP, UMP, dTMP values.

Table 11

B3LYP/6-31g** Calculations of the $\sigma_{\text {iso }}$ in ppm, for Atoms in CMP, UMP, dTMP

\begin{tabular}{|l|c|c|c|c|}
\hline Atom & Method & CMP & UMP & dTMP \\
\hline O2 & GIAO & -514.3770 & -513.0544 & $\sigma_{\text {iso }}$ \\
& CSGT & -511.8219 & -510.5122 & -14.0452 \\
& GIAO & 366.2695 & 366.4302 & -0.1908 \\
O1 & CSGT & 293.7501 & 293.8301 & 293.1990 \\
& GIAO & -590.5471 & -587.5774 & -720.9073 \\
O3 & CSGT & -587.4588 & -584.3941 & -722.2328 \\
& GIAO & 15.5493 & 12.7500 & -535.5780 \\
O5' & CSGT & 30.8019 & 27.9149 & -535.5553 \\
& GIAO & 233.7576 & 233.6205 & 232.8569 \\
C5' & CSGT & 239.4151 & 239.0236 & 239.5009 \\
& GIAO & 125.1186 & 125.0175 & 125.8444 \\
& CSGT & 125.0595 & 24.9494 & 125.8703 \\
\hline
\end{tabular}




\begin{tabular}{|l|c|c|c|c|}
\hline C4' & GIAO & 100.5310 & 100.3551 & 106.8920 \\
C3' & CSGT & 102.2623 & 102.0066 & 108.0079 \\
C2' & GIAO & 121.1567 & 120.5738 & 117.1316 \\
& CSGT & 120.2270 & 119.7958 & 116.3437 \\
C1' & GIAO & 121.6867 & 119.7113 & 152.4803 \\
& CSGT & 119.1631 & 117.3912 & 154.0507 \\
O1' & GIAO & 95.1572 & 97.5965 & 99.9643 \\
& CSGT & 94.8540 & 97.3514 & 100.2233 \\
N1 & GIAO & 240.3374 & 245.0119 & 230.6627 \\
& CSGT & 232.8270 & 237.3346 & 224.5652 \\
C2 & GIAO & 132.1447 & 136.7890 & 129.6839 \\
O7 & CSGT & 128.9853 & 134.3059 & 126.7020 \\
& GIAO & 39.9668 & 45.6711 & 45.8944 \\
N3 & CSGT & 34.8292 & 39.1514 & 39.9325 \\
& GIAO & 14.3922 & 40.1028 & 62.3734 \\
C4 & CSGT & 8.9886 & 39.7758 & 60.8320 \\
& GIAO & 67.9164 & 113.2957 & 118.0751 \\
& CSGT & 60.5725 & 106.7640 & 110.5459 \\
& GIAO & 48.8156 & 45.0549 & 49.0805 \\
& CSGT & 44.0677 & 39.2903 & 42.6477 \\
& GIAO & 109.6685 & 110.6851 & 109.0991 \\
& CSGT & 104.8438 & 105.6880 & 105.4772 \\
& GIAO & 94.6256 & 98.9236 & 99.1797 \\
& CSGT & 92.0962 & 96.4334 & 93.7506 \\
& & & 46.4841 & 79.9393 \\
& & & 41.6251 & 71.4099 \\
\hline
\end{tabular}


Table 12

Relative (to TMS) Shifts in ppm for ${ }^{13} \mathrm{C}-\mathrm{NMR}$ and $1 \mathrm{H}-\mathrm{NMR}$ of CMP, UMP, dTMP using GIAO and CSGT Method at B3LYP/6-31 $\mathrm{g}^{* *}$. The NMR Chemical Shift $\left(\delta=\sigma_{\text {iso }}\right.$ TMS- $\sigma_{\text {iso }}$ Sample).

\begin{tabular}{|c|c|c|c|c|}
\hline Atom & Method & CMP & UMP & dTMP \\
\hline \multirow[t]{2}{*}{$\mathrm{C} 5^{\prime}$} & GIAO & 66.7458 & 66.8469 & 66.0200 \\
\hline & CSGT & 68.0156 & 68.125 & 67. 2048 \\
\hline \multirow[t]{2}{*}{$\mathrm{C} 4^{\prime}$} & GIAO & 91.3334 & 91.5093 & 84.9724 \\
\hline & CSGT & 90.8128 & 91.0685 & 85.0672 \\
\hline \multirow[t]{2}{*}{ C3' } & GIAO & 70.7077 & 71.2906 & 74. 7328 \\
\hline & CSGT & 72.8481 & 73. 2793 & 76. 7314 \\
\hline \multirow[t]{2}{*}{$\mathrm{C} 2^{\prime}$} & GIAO & 70.1777 & 72. 1531 & 39. 3841 \\
\hline & CSGT & 73. 9120 & 75.6839 & 39.0244 \\
\hline \multirow[t]{2}{*}{$\mathrm{Cl}^{\prime}$} & GIAO & 96.7072 & 94.2679 & 91.9001 \\
\hline & CSGT & 98.2211 & 95.7237 & 92.8518 \\
\hline \multirow[t]{2}{*}{$\mathrm{C} 2$} & GIAO & 151.8976 & 146. 1933 & 145.9700 \\
\hline & CSGT & 158. 2459 & 153. 9237 & 153. 1426 \\
\hline \multirow[t]{2}{*}{$\mathrm{C} 4$} & GIAO & 143.0488 & 146.8095 & 142. 7839 \\
\hline & CSGT & 149.0074 & 153. 7843 & 150.4274 \\
\hline \multirow[t]{2}{*}{$\mathrm{C} 5$} & GIAO & 82. 1959 & 81.1793 & 82.7653 \\
\hline & CSGT & 88. 2313 & 87.3871 & 87.5979 \\
\hline \multirow[t]{2}{*}{$\mathrm{C} 6$} & GIAO & 97. 2388 & 92.9408 & 92.6847 \\
\hline & CSGT & 100. 9789 & 96.6417 & 99. 3245 \\
\hline \multirow[t]{2}{*}{ H6 } & GIAO & 4. 1373 & 3. 8745 & 4. 0302 \\
\hline & CSGT & 1. 914 & 1.8724 & 1. 9107 \\
\hline \multirow[t]{2}{*}{$\mathrm{H} 5$} & GIAO & 3. 7528 & 3. 2124 & \\
\hline & CSGT & 1.363 & 0.8072 & \\
\hline \multirow[t]{2}{*}{$\mathrm{H} 1^{\prime}$} & GIAO & 3. 6604 & 3. 7883 & 4. 3442 \\
\hline & CSGT & 1.6391 & 1. 8535 & 2. 1017 \\
\hline \multirow[t]{2}{*}{$\mathrm{H} 5^{\prime}$} & GIAO & 3. 5612 & 3. 5541 & 3.5052 \\
\hline & CSGT & 1. 6425 & 1. 6358 & 1. 7646 \\
\hline \multirow[t]{2}{*}{ H5 } & GIAO & 3. 5662 & 3. 5665 & 3. 7272 \\
\hline & CSGT & 1. 2322 & 1. 1845 & 1. 8797 \\
\hline
\end{tabular}


Table 13

B3LYP/6-31g** Calculations of the $\sigma_{\text {iso }}$ and $\eta$ in ppm for Atoms in Mg-Nucleotides of CMP, UMP, dTMP

\begin{tabular}{|c|c|c|c|c|c|c|c|}
\hline \multirow{2}{*}{ Atom } & \multirow{2}{*}{ Method } & \multicolumn{2}{|c|}{$\mathrm{Mg}(\mathrm{H} 2 \mathrm{O}) 4 \mathrm{CMP}$} & \multicolumn{2}{|c|}{$\mathrm{Mg}(\mathrm{H} 2 \mathrm{O}) 5 \mathrm{UMP}$} & \multicolumn{2}{|c|}{$\mathrm{Mg}(\mathrm{H2O}) 5$ dTMP } \\
\hline & & $\sigma_{\text {iso }}$ & $\eta$ & $\sigma_{\text {iso }}$ & $\eta$ & $\sigma_{\text {iso }}$ & $\eta$ \\
\hline \multirow[t]{2}{*}{$\mathrm{O} 2$} & GIAO & 226.5585 & -46.9672 & 224. 1038 & 3. 5428 & 218.7024 & 4. 3389 \\
\hline & CSGT & 245.7534 & -2.9947 & 244. 7279 & 3. 6289 & 236.7522 & 11.228 \\
\hline \multirow[t]{2}{*}{$\mathrm{P}$} & GIAO & 386.6372 & -0.5633 & 385.6625 & 19.2799 & 382.4083 & 3. 2854 \\
\hline & CSGT & 325.6397 & -0.5702 & 325.3632 & -2086.1338 & 327.6836 & 3. 9113 \\
\hline \multirow[t]{2}{*}{$\mathrm{O} 1$} & GIAO & 197.3560 & 2. 0219 & 197.0063 & -0.1012 & 215.0692 & 4. 0250 \\
\hline & CSGT & 210.9127 & 4. 7907 & 212. 1973 & 0.0291 & 227.2497 & 1. 2191 \\
\hline \multirow[t]{2}{*}{ O3 } & GIAO & 215.5189 & -0.3773 & 219.6031 & 2. 2073 & 196. 0184 & -115.6696 \\
\hline & CSGT & 232.8156 & 0.6239 & 236. 2105 & 1. 2732 & 211.6728 & -13.1598 \\
\hline \multirow[t]{2}{*}{ O5' } & GIAO & 244.2142 & -3.5982 & 240.129 & -8.5761 & 245.8752 & 1. 1966 \\
\hline & CSGT & 245.0896 & -2.3198 & 242.0614 & -5.2685 & 250.4434 & 0.5286 \\
\hline \multirow[t]{2}{*}{$\mathrm{C} 5^{\prime}$} & GIAO & 130.4883 & -17.5763 & 131.7728 & -7.2811 & 128.0928 & -2.3850 \\
\hline & CSGT & 129.0078 & 11. 2064 & 130.3865 & -11.7384 & 127.9573 & -2.2781 \\
\hline \multirow[t]{2}{*}{$\mathrm{C} 4^{\prime}$} & GIAO & 108.5125 & 1. 6408 & 108. 3150 & 1. 1493 & 103. 1658 & -26.0886 \\
\hline & CSGT & 108. 7888 & 1. 4643 & 108. 1458 & 1. 1457 & 104. 3985 & 69.0538 \\
\hline \multirow[t]{2}{*}{$\mathrm{C} 3^{\prime}$} & GIAO & 120.0705 & 0.8833 & 123. 2455 & 0.9566 & 118.0323 & 2. 2854 \\
\hline & CSGT & 117.9747 & 0.7957 & 120.9372 & 0.82990 & 117.5202 & 2. 1381 \\
\hline \multirow[t]{2}{*}{$\mathrm{C} 2^{\prime}$} & GIAO & 119. 9059 & -0.0243 & 119.6933 & 0.9584 & 156. 9011 & -5.9778 \\
\hline & CSGT & 117. 9733 & -0.0026 & 117. 7392 & 1. 1354 & 157.9196 & -7.2084 \\
\hline \multirow[t]{2}{*}{$\mathrm{C} 1^{\prime}$} & GIAO & 92.3321 & -1.40396 & 94.8099 & -1.2215 & 97.7546 & -6.2002 \\
\hline & CSGT & 91.6214 & -1.2893 & 94.4952 & -1.0589 & 97.1814 & -5.1565 \\
\hline \multirow[t]{2}{*}{ O1' } & GIAO & 256.4880 & 0.4694 & 250.8207 & 0.2404 & 246. 4044 & -1.4148 \\
\hline & CSGT & 250.0488 & 0.4707 & 245.8660 & 0.2418 & 239.8925 & -1.2924 \\
\hline \multirow[t]{2}{*}{ N1 } & GIAO & 101.0952 & 54. 2223 & 117. 2015 & -6.0647 & 117.0489 & 0.6819 \\
\hline & CSGT & 97.8561 & -54.9369 & 113. 1909 & -5.0327 & 113.5592 & 0.5794 \\
\hline \multirow[t]{2}{*}{$\mathrm{C} 2$} & GIAO & 49.0915 & 70.7041 & 53.7386 & -2.8224 & 57.7475 & -1.7563 \\
\hline & CSGT & 42.8417 & -111.0038 & 47.6462 & 2. 8930 & 48. 5867 & -1.7628 \\
\hline \multirow[t]{2}{*}{ O7 } & GIAO & 32.2392 & -8.8977 & 38. 3589 & -1.6768 & 40. 0617 & 0.2607 \\
\hline & CSGT & 26.8208 & -8.5985 & 36.1594 & 1. 6072 & 36.5339 & 0.1914 \\
\hline \multirow[t]{2}{*}{ N3 } & GIAO & 59.0252 & -8.7095 & 108.1047 & 0.3558 & 107.1225 & -4.1925 \\
\hline & CSGT & 52.0032 & 10.6351 & 101.8760 & 0.3222 & 101.0808 & -3.5567 \\
\hline \multirow[t]{2}{*}{$\mathrm{C} 4$} & GIAO & 38.7826 & -5.2799 & 44. 9328 & 0.4644 & 43. 1285 & 0.4340 \\
\hline & CSGT & 33.8865 & -5.4036 & 39.2421 & 0.3676 & 37.5025 & 0.3913 \\
\hline \multirow[t]{2}{*}{$\mathrm{C} 5$} & GIAO & 106. 1726 & 0.04226 & 94.6694 & 0.7960 & 86.8756 & 0.2144 \\
\hline & CSGT & 100.4281 & -0.0926 & 88.6740 & -0.8128 & 81.9921 & 0.1228 \\
\hline
\end{tabular}




\begin{tabular}{|l|l|l|l|l|l|l|c|}
\hline C6 & GIAO & 51.6989 & -0.8031 & 56.1445 & -0.4309 & 58.3169 & -5.1678 \\
& CSGT & 47.5190 & -0.8601 & 51.8109 & -0.4227 & 53.3347 & -1.1616 \\
& GIAO & & & -53.3664 & 5.2413 & -35.2001 & 0.4340 \\
& CSGT & & & -58.6325 & 4.7164 & -40.6903 & 0.2002 \\
\hline
\end{tabular}

Table 14

Relative (to TMS) Shifts in ppm for 13C-NMR and 1H-NMR of CMP, UMP, dTMP using GIAO and CSGT Method at B3LYP/6-31g**. The NMR Chemical Shift ( $\delta=\sigma_{\text {iso }}$ TMS- $\sigma_{\text {iso }}$ Sample)

\begin{tabular}{|c|c|c|c|c|}
\hline Atoms & Method & $\mathrm{Mg}(\mathrm{H} 2 \mathrm{O}) 4 \mathrm{CMP}$ & $\mathrm{Mg}(\mathrm{H} 2 \mathrm{O}) 5 \mathrm{UMP}$ & $\mathrm{Mg}(\mathrm{H} 2 \mathrm{O}) 5 \mathrm{dTMP}$ \\
\hline \multirow[t]{2}{*}{$\mathrm{C} 5^{\prime}$} & GIAO & 61.3761 & 60.0916 & 63.7712 \\
\hline & CSGT & 64.0673 & 62.6886 & 64.9823 \\
\hline \multirow[t]{2}{*}{$\mathrm{C} 4^{\prime}$} & GIAO & 83.3519 & 83.5494 & 88.6986 \\
\hline & CSGT & 84. 2863 & 84. 9293 & 88.6766 \\
\hline \multirow[t]{2}{*}{$\mathrm{C}^{\prime}$} & GIAO & 71.7939 & 68.6189 & 73.8321 \\
\hline & CSGT & 75.1004 & 72.1379 & 75.5549 \\
\hline \multirow[t]{2}{*}{$\mathrm{C} 2^{\prime}$} & GIAO & 71.9585 & 72. 1711 & 34.9633 \\
\hline & CSGT & 75. 1018 & 75.3359 & 35.1555 \\
\hline \multirow[t]{2}{*}{$\mathrm{C} 1^{\prime}$} & GIAO & 99.5323 & 97.0545 & 94. 1098 \\
\hline & CSGT & 101.4537 & 98.5799 & 95.8937 \\
\hline \multirow[t]{2}{*}{$\mathrm{C} 2$} & GIAO & 142. 7729 & 138. 1258 & 134. 1169 \\
\hline & CSGT & 143.9836 & 145.4289 & 144. 4884 \\
\hline \multirow[t]{2}{*}{$\mathrm{C} 4$} & GIAO & 153. 0818 & 146.9316 & 148.7359 \\
\hline & CSGT & 159. 1886 & 153.8330 & 155.5726 \\
\hline \multirow[t]{2}{*}{ C5 } & GIAO & 85.6918 & 97.1950 & 104. 9888 \\
\hline & CSGT & 92.6470 & 104. 4011 & 111.0830 \\
\hline \multirow[t]{2}{*}{ C6 } & GIAO & 140.1655 & 135.7199 & 133.5475 \\
\hline & CSGT & 145.5561 & 141.2642 & 139. 7404 \\
\hline \multirow[t]{2}{*}{ H6 } & GIAO & 6.8675 & 6. 5400 & 6. 2692 \\
\hline & CSGT & 4.5447 & 4. 2703 & 3. 9418 \\
\hline \multirow[t]{2}{*}{ H5 } & GIAO & 5. 0146 & 5. 1211 & \\
\hline & CSGT & 2. 8509 & 2. 8774 & \\
\hline \multirow[t]{2}{*}{$\mathrm{HI}^{\prime}$} & GIAO & 4. 2588 & 4. 1879 & 4. 8331 \\
\hline & CSGT & 2. 0505 & 1. 9026 & 2. 7834 \\
\hline \multirow[t]{2}{*}{ H5' } & GIAO & 3. 7881 & 3. 8469 & 3. 1857 \\
\hline & CSGT & 2. 0462 & 2. 0789 & 1. 7267 \\
\hline \multirow[t]{2}{*}{ H5" } & GIAO & 3. 6275 & 3. 3817 & 3. 4009 \\
\hline & CSGT & 1.7449 & 1.7436 & 1.5303 \\
\hline
\end{tabular}


Table 15

HF/6-31g** Calculations of the $\sigma_{\text {iso }}$ in ppm for Atoms in Metalated or Nonmetalated CMP, UMP, dTMP (Charge and Multipilicity -2,3)

\begin{tabular}{|c|c|c|c|c|c|c|}
\hline Atom & CMP & $\mathrm{Mg}(\mathrm{H} 2 \mathrm{O}) 4 \mathrm{CMP}$ & UMP & $\mathrm{Mg}(\mathrm{H} 2 \mathrm{O}) 5 \mathrm{UMP}$ & dTMP & Mg(H2O)5dTMP \\
\hline $\mathrm{C}^{\prime}$ & 145.7379 & 146.0515 & 145.7585 & 147.2284 & 144.6637 & 142.7135 \\
\hline $\mathrm{C}^{\prime}$ & 125.0030 & 126.0659 & 124.2624 & 125.3747 & 116.5172 & 122.3503 \\
\hline $\mathrm{C}^{\prime}$ & 138.5291 & 136.9841 & 138. 7352 & 139.6812 & 139. 4666 & 135.8682 \\
\hline $\mathrm{C} 2^{\prime}$ & 136.5700 & 135.8006 & 135.2260 & 135.6797 & 168. 1820 & 169. 3802 \\
\hline $\mathrm{C} 1^{\prime}$ & 114.3494 & 112.2896 & 115. 4666 & 114. 7350 & 125.1421 & 117.5060 \\
\hline C6 & 17.7335 & 48.3862 & 22.5274 & 59. 4509 & -2.8103 & 58.9970 \\
\hline $\mathrm{C} 4$ & 20.8724 & 32.9057 & 35.1250 & 45.3533 & 46.5828 & 42.6675 \\
\hline $\mathrm{C} 5$ & 105.0896 & 117.7746 & 90.2017 & 106.07750 & 58.1032 & 100.2425 \\
\hline $\mathrm{C} 2$ & 46.5906 & 49. 1639 & 52.6562 & 54.3845 & 58.28024 & 55.2619 \\
\hline $\mathrm{H} 1^{\prime}$ & 28.5188 & 28. 1328 & 29.0910 & 29.0370 & 28.6257 & 27.8413 \\
\hline $\mathrm{H} 6$ & 22.8798 & 24.6581 & 23. 0883 & 25.1749 & 22. 0930 & 25.4905 \\
\hline H5 & 26.6215 & 26.9912 & 26.4179 & 26.8249 & & \\
\hline $\mathrm{H}^{\prime}$ & 28.8930 & 28.8101 & 28.8735 & 28.9776 & 29. 7987 & 29.0149 \\
\hline H5" & 28.3595 & 28.5846 & 28. 3058 & 28. 4569 & 28.6257 & 28.9690 \\
\hline N8 & 219.2447 & 197.7547 & & & & \\
\hline N1 & 168.1530 & 128.8930 & 170.4110 & 147.8460 & 159.7488 & 148. 1081 \\
\hline$N ?$ & & & & & & \\
\hline N3 & 124. 1017 & 81.8900 & 149. 9611 & 132. 6250 & 136. 2581 & 131.7112 \\
\hline $\mathrm{O} 3$ & -399.1824 & 244.3538 & -3373.0419 & 248. 1015 & 252. 1960 & 227.5930 \\
\hline $\mathrm{O} 2$ & 26. 1615 & 255.0413 & 263.5201 & 253. 7420 & 245.0883 & 250.5244 \\
\hline O1 & 279.8879 & 230.3126 & 279.8830 & 229.6790 & 256.7207 & 244.6401 \\
\hline O5' & 284.4205 & 281.5102 & 284.3592 & 278. 3393 & 276.9306 & 280.5709 \\
\hline O1' & 289.7209 & 295.0279 & 291.1317 & 291.301 & 286.6974 & 286. 9013 \\
\hline 07 & 100.6183 & 62.7614 & 109.6324 & 63.3416 & 42. 2072 & 63. 6221 \\
\hline $\mathrm{O} 8$ & & & 131.8189 & -36.5383 & -50.8827 & -20.0441 \\
\hline $\mathrm{P}$ & 421.4834 & 422.8997 & 421.8483 & 422.4350 & 415. 2184 & 421.3731 \\
\hline
\end{tabular}


Table 16

Relative (to TMS) Shifts in ppm for ${ }^{13} \mathrm{C}-\mathrm{NMR}$ and ${ }^{1} \mathrm{H}-\mathrm{NMR}$ of Nucleotides using GIAO Method at HF/6-31g**. The Chemical Shifts ( $\delta=\sigma_{\text {iso }}$ TMS $-\sigma_{\text {iso }}$ Sample)

\begin{tabular}{|c|c|c|c|c|c|c|}
\hline Atom & CMP & $\mathrm{Mg}(\mathrm{H} 2 \mathrm{O}) 4 \mathrm{CMP}$ & UMP & $\mathrm{Mg}(\mathrm{H} 2 \mathrm{O}) 5 \mathrm{UMP}$ & dTMP & $\mathrm{Mg}(\mathrm{H} 2 \mathrm{O}) 5 \mathrm{dTMP}$ \\
\hline $\mathrm{C} 5^{\prime}$ & 57. 4169 & 57.1033 & 57.3963 & 55.9264 & 58.4911 & 60.4413 \\
\hline $\mathrm{C} 4^{\prime}$ & 78. 1518 & 77.0889 & 78.8924 & 77. 7801 & 86.6376 & 80.8045 \\
\hline $\mathrm{C}^{\prime}$ & 64.6257 & 66.1707 & 64.4196 & 63.4736 & 63.6882 & 67.2866 \\
\hline $\mathrm{C} 2^{\prime}$ & 66.5848 & 67.3542 & 67.9288 & 67.4751 & 34. 9728 & 33.7746 \\
\hline $\mathrm{C}^{\prime}$ & 88.8054 & 90.8652 & 87.6882 & 88.4198 & 78.0127 & 85.6488 \\
\hline C6 & 185.4213 & 154.7686 & 180.6274 & 146.7039 & 205.9651 & 144. 1578 \\
\hline C5 & 98.0652 & 85.3802 & 112.9531 & 97.0773 & 145.0516 & 102.9123 \\
\hline $\mathrm{C} 4$ & 182. 2824 & 170.2491 & 168.0298 & 157. 8015 & 198.5720 & 160.4873 \\
\hline $\mathrm{C} 2$ & 156.5642 & 153. 9909 & 150.4986 & 148.7703 & 144.8724 & 147.8929 \\
\hline H6 & 9. 4562 & 7. 6779 & 9. 2477 & 7. 1611 & 10.2430 & 6.8455 \\
\hline H5 & 5. 7145 & 5.3448 & 5. 9181 & 5.5111 & & \\
\hline $\mathrm{H} 1^{\prime}$ & 3. 8172 & 4. 2032 & 3. 2450 & 3. 2990 & 3.7103 & 4. 4947 \\
\hline $\mathrm{H} 5^{\prime}$ & 3. 443 & 3.5259 & 3. 4625 & 3. 3584 & 2. 5373 & 3. 3211 \\
\hline H5" & 3. 9765 & 3. 7514 & 4. 0302 & 3. 8791 & 3. 7103 & 3. 3670 \\
\hline
\end{tabular}

\section{REFERENCES:}

1. M.S. Zetter, G.Y. -S. Lo, H.W. Dodgen and J.P. Hunt, J. Am. Chem. Soc., 100, 4430-4436 (1978).

2. J.A. Happe and M. Morales, J. Am. Chem. Soc., 88, 2077-2078 (1966).

3. A. Tissieres, J.D. Watson, D. Schlessinger and B.R. Hollingworth, J. Mol. Biol., 1, 221 (1959).

4. W.E.C. Wacker and B.L. Vallee, J. Biol. Chem., 234, 3257 (1959).

5. K. Aoki, Met. Ions Biol. Syst., 32, 91-134 (1996).

6. D.B. Davies, P. Rajani and H. Sadikot, J. Chem. Soc. Perkin Trans., 1958 (2), 279-285.

7. R.B. Martin and Y.H. Mariam, Met. Ions Biol. Syst., 8, 57-124 (1979).

8. R. Tribolet and H. Sigel, Eur. J. Biochem., 163, 353-363 (1978).

9. R.M. Izatt, J.J. Christensen and J.H. Rytting, Chem. Rev., 71, 439 (1971).

10. A.T. Tu and M.J. Heller, in: Metal Ions in Biological Systems, Vol. 1, H. Sigel (Ed.), Dekker, New York, 197; p. 2.

11. N.C. Li, R. Scruggs and E.D. Becker, J. Am. Chem. Soc., 84, 4650 (1962).

12. L.D. Barron, Molecular Light Scattering Optical Activity, Cambridge University Press, Cambridge, 1982.

13. P. Bour, V. Sychrovsky, P. Malon, J. Hanzlikova, V. Baumruk, J. Pospisek and M. Budesinsky, J. Phys. Chem. A, 106, 7321-73227 (2002). 
15. a) K. Wolinski, J.F. Hilton and P. Pulaj, J. Am. Chem. Soc., 112, 8251 (1990); b) L. Dodds, R. Cweeny and A. Sadlej, J. Mol. Phys., 41, 1419 (1980); c) R. Ditchfield, Mol. Phys., 27, 789 (1974); d) R. Mcweeny, Phys. Rev., 126, 1024 (1962); e) F. London, J. Phys. Radium (Paris), 8, 397 (1937).

16. J.R. Cheeseman, M.J. Frisch, G.W. Trucks and T.A. Keith, J. Chem. Phys., 104, 5497 (1996).

17. M.J. Frisch, J.E. DelBene, J.S. Binkley and H.F. Schaefer III, J. Chem. Phys. 84, 2279 (1986).

18. NBO Method (a) E.D. Glendening, A.E. Reed, J.E. Carpenter and F. Weinhold, NBO Version 3.1. (b) E.D. Glendening and F. Weinhold, J. Comput. Chem., 19, 628 (1998) and references therein.

19. P.C. Hariharan and J.A. Pople, Theor. Chim., 28, 213 (1973).

20. B.A. Hathaway, G. Day, M. Lewis and R. Glaser, J. Chem. Soc. Perkin Trans., 1998 (2), 2713-2719 and references therein.

21. L.C. Yang and R.G. Parr, Phys. Rev. B, 37, 785 (1988).

22. B. Miehlich, A. Savin, H. Stoll and H. Preuss, Chem. Phys. Lett., 157, 200 (1989).

23. A.D. Becke, J. Chem. Phys., 98, 5648 (1993).

24. K.M. Al-Fouti, J.R. Hanson and P.B. Hitchcock, J. Chem. Research (S), 2002, 624.

25. R.M. Appel, Angew. Chem. Int. Ed. Engl., 14, 801 (1975).

26. F.S. Boys and F. Bernardi, Mol. Phys., 19, 553 (1970).

27. S.S. Massoud and H. Siugel, Inorg. Chem., 24, 1447 (1988).

28. a) H. Brintzinger, Helv. Chim. Acta, 48, $47-54$ (1965), (b) H. Brintzinger and G. Hammes, Inorg. Chem., 5, 1286-1287 (1966).

29. T. Theophanides and H.A. Tajmir-Riahi, NATO ASI Ser. C, 139, 137-152 (1984).

30. H.A. Tajmir-Riahi and T. Theophanides, Inorg. Chim. Acta, 80, 183 (1983).

31. U. Thewalt, C.E. Bugg and R.E. Marsch, Acta Crystallogr., Sect. B, B26, 1089 (1970).

32. C. Altona and M. Sundaralingam, J. Am. Chem. Soc., 94, 8205 (1972).

33. M. Sundaralingam, Ann. N.Y. Acad. Sci., 3, 255 (1975).

34. M. Sundaralingam, Biopolymers, 7, 821-860 (1969).

35. $\chi_{\mathrm{CN}}$ in the definition of Saenger $/ 36 /$ corresponds to $\Phi_{\mathrm{CN}}$ in the definition of Donohue and Trueblood $137 \%$.

36. W. Saenger, Angew. Chem., Int. Ed., Engl., 12, 591 (1973); Angew Chem., 85, 680 (1973).

37. J. Donohue and K.N. Trueblood, J. Mol. Biol., 2, 363 (1960).

38. V. Swaminathan and M. Sundaralingam, CRC Rev. Biochem., 6, 245 (1979).

39. M.J. Frisch et al., Gaussian 98, Revision A9, Gaussian Inc., Pittsburgh, PA, 1998.

40. I. Vickovic, Lecture. The Structure of DNA and RNA. The Double Helix. University of Zagreb, Faculty of Science, 2003.

41. U. Haeberlen, Adv. Magn. Reson. Suppl., 1, 1 (1976). 
